\section{Câncer de mama feminino: aspectos clínicos e patológicos dos casos cadastrados de 2005 a 2008 num serviço público de oncologia de Sergipe}

\section{Female breast cancer: clinical and pathological aspects of cases registered between 2005 and 2008 at a public oncology service in the Brazilian State of Sergipe}

Leila Luíza Conceição Gonçalves 1

Simone Barbosa Santos 2

Emily Carvalho Marinho 3

Ana Maria de Almeida 4

Alessandro Henrique da Silva Santos 5

Ângela Maria Melo Sá Barros 6

Ricardo Fakhouri 7

1-3 Departamento de Enfermagem. Universidade Federal de Sergipe. Cidade Universitária Prof. José Aloísio de Campos. Av. Marechal Rondon, s.n. Jardim Rosa Elze. São Cristóvão. Aracaju, SE, Brasil. CEP: 49.100-000: E-mail: leilaluiza@globo.com

4 Escola de Enfermagem de Ribeirão Preto. Universidade de São Paulo. Ribeirão Preto, São Paulo, Brasil.

5 Núcleo de Estatística. Universidade Federal de Sergipe. Aracaju, Sergipe, Brasil.

6 Clínica Oncohematos Ltda. Aracaju, Sergipe, Brasil.

7 Departamento de Medicina. Universidade Federal de Sergipe. Aracaju, Sergipe, Brasil.

\section{Resumo}

Objetivos: conhecer os aspectos clinicos e patológicos dos casos de câncer de mama feminino cadastrados num serviço público de oncologia de Sergipe.

Métodos: procurou-se fazer uma associação do estadiamento com as características presentes nos tumores. A coleta de dados foi realizada em 165 prontuários de mulheres cadastradas no período de 2005 a 2008 no ambulatório de oncologia do Hospital Cirurgia, localizado em Aracaju-Sergipe, por meio de formulário de coleta de dados, em 2009.

Resultados: identificou-se o carcinoma ductal invasivo como o tipo de neoplasia mamária mais frequente com 80,8\% dos casos; 76 (46,1\%) neoplasias foram diagnosticadas em estádio avançado (IIB, III e IV) e a mastectomia prevaleceu como tipo de cirurgia utilizado no tratamento independente do estadiamento. Quanto aos receptores hormonais todos apresentaram associação com o grau do estádio e, ainda, notou-se que a positividade do estrogênio e progesterona é fator de proteção para um pior prognóstico.

Conclusão: é notável que os casos de câncer de mama estudados apresentam maior agressividade biológica, cursando com pior prognóstico, considerando o estádio clínico. Fazem-se necessárias ações de detecção precoce voltadas ao câncer de mama na atenção básica de Sergipe para redução da morbi-mortalidade, melhora da sobrevida e qualidade de vida entre as mulheres acometidas por essa neoplasia.

Palavras-chave Neoplasias da mama, Patologia clínica, Oncologia 


\section{Introdução}

Entre o público feminino uma das doenças mais temidas é o câncer de mama, principalmente, na faixa etária após os 35 anos de idade na qual sua incidência cresce rápida e progressivamente. ${ }^{1}$

Associado a esse fator está o diagnóstico tardio da doença, ocasionado por uma política ineficaz de controle e rastreamento do câncer; que tem na mamografia, aliada ao exame clínico das mamas e ao auto-exame, seus instrumentos fundamentais. 1,2

Para o ano de 2010 foram esperados 49.240 casos novos de câncer de mama no Brasil, com um risco estimado de 49 casos para cada 100 mil mulheres. No Estado de Sergipe, para o mesmo ano, estimou-se 340 novos casos; desses, 160 foram previstos ocorrerem em Aracaju, capital do Estado. ${ }^{3}$

A Organização Mundial da Saúde (OMS) adverte que, para se ter controle eficaz do câncer de mama, são necessárias medidas que garantam um diagnóstico da doença nas fases iniciais, ressaltando, assim, a importância das ações de detecção precoce. De fato essas estratégias de prevenção reduzem, significativamente, a mortalidade por câncer de mama e as repercussões físicas, psíquicas e sociais causadas por esse tipo de neoplasia. ${ }^{3}$

Os carcinomas invasivos ductais e lobulares são as formas mais comuns de câncer de mama, que se tornam evidentes com os dados de prevalência, os quais apontam que o carcinoma ductal invasivo (CDI) varia de $50-75 \%$ e, a do carcinoma lobular invasivo (CLI), de $5-15 \%$ de todas as neoplasias invasivas da mama. 4 Outros tipos mais raros de câncer de mama invasivos são: carcinoma medular, carcinoma mucinoso, carcinoma papilífero, carcinoma inflamatório.5-7

Apesar dos tumores malignos apresentarem uma grande variedade, eles possuem um comportamento biológico semelhante, quanto ao crescimento, invasão local, destruição dos órgãos vizinhos, disseminação regional e sistêmica. ${ }^{8}$

A União Internacional Contra o Câncer (UICC) classifica a evolução das neoplasias malignas para definir a melhor terapêutica e a sobrevida dos pacientes. Sendo assim, a classificação para o estadiamento clínico (TNM) e patológico (pTNM) para os tumores sólidos, como o da mama, segundo a UICC, fundamenta-se na análise do tamanho do nódulo em sua maior dimensão $(\mathrm{T})$, ausência ou presença de metástase linfonodos regionais $(\mathrm{N})$ e ausência ou presença de mestástase a distância (M) apenas para os tumores histologicamente confirmados. 9

Para que a avaliação dos aspectos patológicos do câncer de mama seja realizada é necessária a implementação do tratamento cirúrgico. Durante a realização da cirurgia a retirada do tumor primário é realizada em blocos e, pelo câncer de mama se caracterizar como uma doença sistêmica tornou-se mandatório a dissecção axilar completa homolateral que, possivelmente, também estaria acometida por células neoplásicas. ${ }^{9}$ Atualmente, a dissecção axilar completa deve ser realizada quando extremamente necessário após avaliação minuciosa do caso, a fim de evitar mutilação e danos funcionais desnecessários.

A avaliação do comprometimento linfático, também pode ser feita por meio da técnica que utiliza o linfonodo sentinela (LS). A aplicação desta técnica, identificando o não comprometimento do primeiro linfonodo por células cancerígenas da rede linfática proximal do tumor primário, está associado a uma melhor preservação do funcionamento do braço, menor morbidade e uma melhor qualidade de sobrevida. 10 Contrariamente, quando existe comprometimento do LS, há necessidade da dissecção axilar completa.

Alguns fatores prognósticos para o câncer de mama relacionam-se principalmente a: aspectos anatômicos (tamanho do tumor, comprometimento de linfonodos e subtipos histológicos); características patológicas quantitativas (grau histológico, necrose tumoral e índice mitótico); responsividade endócrina (receptores hormonais de estrogênio e progesterona); fatores moleculares específicos reguladores do crescimento tumoral (oncogenes e genes supressores tumorais); e fatores preditivos de metástases peculiares para os diferentes órgãos. 11

Cerca de dois terços das neoplasias de mama expressam a proteína receptora para hormônios, sendo, então, considerados positivos para receptores hormonais (Rh). ${ }^{1}$ Dessa forma, recomenda-se para pacientes com diagnóstico de câncer de mama a realização da pesquisa desses receptores, sendo os mais comuns os receptores de estrogênio (RhE) e os receptores de progesterona (RhP). ${ }^{9}$ Outro marcador que também é extensamente estudado e deve ser investigado nos casos de câncer de mama é o C-erbB-2 que revela um prognóstico sombrio, exigindo uma análise cuidadosa na implementação da terapêutica. 12

No que diz respeito ao grau histológico, o método de avaliação mais utilizado é o de Nottingham, que corresponde ao sistema de graduação de Scarff, Bloom, Richardson, 13 modificado por Elston e Ellis. O método classifica o grau histológico em três categorias em três categorias: bem diferenciado (grau I), moderadamente diferenciado (grau II) 
e pouco diferenciado (grau III). São utilizados como parâmetros nessa graduação a formação tubular, o pleomorfismo nuclear e o índice mitótico da neoplasia. ${ }^{13}$

O tamanho e o estádio tumoral são os fatores que mais influenciam na sobrevida das pacientes. ${ }^{14} \mathrm{De}$ uma maneira geral, o bom prognóstico do câncer mamário se dá quando este é detectado precocemente.

Sendo assim, esse estudo teve como objetivos conhecer os aspectos clínicos e patológicos dos casos de câncer mama cadastros no período de 2005 a 2008 num serviço público de oncologia de Sergipe e realizar associação entre estadiamento, tipo de cirurgia realizada, número de linfonodos axilares comprometidos, marcador tumoral C-erbB-2, receptores hormonais (RhE e RhP) e situação do seguimento. A sua importância, foi confirmada ao realizar o levantamento bibliográfico no Lilacs, Medline, SciELO, Pubmed e Elsevier Sciencedirect não sendo encontrado nenhum trabalho com este perfil em Sergipe e por está de acordo com as prioridades do Sistema Único de Saúde, do Plano Nacional de Assistência Integral à Saúde da Mulher e do Pacto pela vida.

Espera-se que esta pesquisa possa contribuir para o conhecimento de profissionais e gestores de saúde quanto aos aspectos clínicos e patológicos da neoplasia mamária no Estado de Sergipe, comparando-os com dados nacionais e internacionais, favorecendo uma melhor elaboração ou aplicação de medidas de detecção precoce, métodos diagnósticos e tratamento, considerando a realidade local.

\section{Métodos}

O estudo foi desenvolvido sob a abordagem retrospectiva, transversal e quantitativa. A coleta dos dados foi feita no ambulatório de oncologia clínica da Fundação Beneficente Hospital Cirurgia coordenado pela Clínica Onco Hematos Ltda no período de abril a outubro de 2009, após avaliação e aprovação do projeto pelo Comitê de Ética em Pesquisa da Universidade Federal de Sergipe através do parecer CAAE de $n^{\circ}$ 1533.0.000. A população foi composta por 165 prontuários de mulheres com câncer de mama, que, realizaram uma biópsia e foram cadastradas na Clínica OncoHematos no período de 2005 a 2008.

Para a obtenção das informações foi construído um formulário de coleta de dados, considerando as seguintes informações relativas ao tumor como: estadiamento, tamanho, classificação do tumor, grau de diferenciação, aspectos anatomopatológicos, tratamento cirúrgico, metástase e seguimento. Estas informações foram coletadas por meio da análise de prontuários, que continham o resultado histopatológico do exame anatomopatológico após cirurgia e imuno-histoquímica, arquivados na instituição acima referida.

Ainda para o desenvolvimento da análise, os casos de câncer de mama foram categorizados em dois grupos de estádio: nível baixo e nível alto. Esta divisão foi baseada nas características do tumor primário, considerando o maior diâmetro do componente invasivo do tumor e da presença de linfonodos positivos relacionados com o risco de recorrência do tumor. ${ }^{5}$ Sendo assim, os tumores primários menores que dois $\mathrm{cm}$ e com linfonodos negativos foram classificados como risco mínimo ou baixo de recorrência e se encontram dentro dos estádios 0 , I e IIA, considerados no estudo como nível baixo. Os tumores primários que apresentaram mais que dois $\mathrm{cm}$ e linfonodos positivos foram classificados como nível elevado de recorrência sendo relativos aos estádios IIB, III e IV, apresentando-se no estudo como nível alto. Após categorização do estádio foi possível associá-lo com os demais fatores. Também, em relação ao número de linfonodos positivos, foi estipulada a seguinte classificação: GP (gânglios positivo) baixo, quando menos de $33 \%$ do total de linfonodos retirados através da dissecção axilar completa eram positivos e GP (gânglios positivo) alto, quando mais de $33 \%$ do total de linfonodos retirados através da dissecção axilar completa eram positivos. Outro fator que foi verificado em associação com o estadiamento foi o marcador C-erbB-2, considerado positivo quando apresentava superexpressão (escore +++ ).

Os dados foram tabulados com o auxílio do software EPI Info, versão 6.04d. Para análise de associação foi utilizado o teste de qui-quadrado para independência em relação ao estádio e tipo de cirurgia, gânglios comprometidos, presença ou não de receptores de estrogênio e progesterona e a presença ou não do marcador C-erbB-2. Para avaliar homogeneidade do tipo de cirurgia e da situação atual do paciente nos grupos de estadiamento baixo e alto do câncer foi utilizado o teste qui-quadrado para homogoneidade. Nas tabelas em que as suposições do teste qui-quadrado não foram satisfeitas, foi utilizado o teste Exato de Fisher. Em todas as conclusões foi considerado o nível de significância de $5 \%$.

\section{Resultados}

Dos 146 prontuários que descreviam o tipo de câncer 
mamário, prevaleceu o carcinoma ductal invasivo (CDI) com 118 casos. Quanto ao grau de diferenciação dos nódulos, 72 (55,8\%), dos 129 casos que possuíam essa informação, apresentavam grau II. Em relação ao estadiamento, tendo como base o sistema TNM, os estádios com maior frequência foram IIA $(34,0 \%)$, seguido do IIIA $(23,3 \%)$, de um total de 150 prontuários que continham essa informação.

A partir dos dados coletados, verificou-se a relação entre o estádio clínico do câncer de mama e tipo de cirurgia realizada e a situação atual da paciente (seguimento) e associação entre estádio clínico número de linfonodos axilares compro- metidos, marcador tumoral C-erbB-2 e receptores hormonais (RhE e RhP).

$\mathrm{O}$ número total de pacientes submetidas à intervenção cirúrgica foi 147 (89\%), dessas 106 (64,2\%) realizaram mastectomia. Verifica-se que tanto no grupo com estadiamento baixo quanto no grupo de estadiamento alto prevaleceu a mastectomia. Porém, através do testes de homogeneidade, a proporção da mastectomia no grupo de estadiamento alto é bem maior do que no grupo de estadiamento baixo $(p<0,001)$, observou-se, ainda que $42(57,2 \%)$ mulheres, diagnosticadas com estádio baixo, foram submetidas à mastectomia (Tabela 1).

Tabela 1

Distribuição da frequência do estádio clínico segundo o tipo de cirurgia e a situação da paciente dos casos de câncer de mama registrados no período de 2005 a 2008 em Aracaju.

\begin{tabular}{|c|c|c|c|c|c|c|c|c|}
\hline \multirow{3}{*}{ Variável } & \multicolumn{4}{|c|}{ Estadiamento do câncer } & & & \multirow{3}{*}{$\mathbf{R R}$} & \multirow{3}{*}{$p$} \\
\hline & \multicolumn{2}{|c|}{ Baixo } & \multicolumn{2}{|c|}{ Alto } & \multicolumn{2}{|c|}{ Total } & & \\
\hline & $\mathrm{n}$ & $\%$ & $\mathrm{n}$ & $\%$ & $\mathrm{n}$ & $\%$ & & \\
\hline \multicolumn{9}{|l|}{ Tipo de cirurgia } \\
\hline Conservadora & 31 & 42,5 & 10 & 13,5 & 41 & 27,9 & 3,14 & $<0,0011$ \\
\hline Mastectomia & 42 & 57,5 & 64 & 86,5 & 106 & 72,1 & - & \\
\hline \multicolumn{9}{|l|}{ Situação atual da paciente } \\
\hline Viva sem evidência de tumor & 63 & 85,1 & 44 & 58,7 & 107 & 71,8 & 1,45 & 0,0012 \\
\hline Viva com evidência de tumor & 2 & 2,7 & 7 & 9,3 & 9 & 6,0 & - & \\
\hline Perda de acompanhamento & 9 & 12,2 & 24 & 32,0 & 33 & 22,1 & - & \\
\hline
\end{tabular}

1 Teste qui-quadrado para homogeneidade; 2 Teste Exato de Fisher.

Também foi observado o seguimento dos casos em estudo, evidenciando que 107 (71,8\%) mulheres seguiam sem evidência de tumor, ou seja, não apresentavam metástase locoregional ou sistêmica e nove mulheres $(6 \%)$ viviam com evidência do tumor, metástase locorregional ou sistêmica.

Verificou-se associação entre a situação atual das mulheres, seguimento, com o estadiamento, pois, 63 $(85,1 \%)$ das mulheres que tiveram a neoplasia classificada em estádio baixo seguiam sem evidências de tumor - o qual possui risco menor de recorrência enquanto, $44(58,7 \%)$ das mulheres que tiveram a neoplasia classificada em estádio alto estavam vivas, mas sem evidência de tumor $(p<0,001)$ (Tabela 1$)$. Ante esses dados, observa-se também que as chances de viver sem evidência de tumor foi de $45,0 \%$ maior entre às mulheres que tiveram a neoplasia classificada em estádio baixo quando comparadas com aquelas classificada em estádio alto.
Entre as mulheres vivas com evidência de tumor seis $(66,7 \%)$ apresentaram metástase à distância (óssea e pulmonar), enquanto três $(33,3 \%)$ apresentaram metástase locorregional. É importante salientar que sete $(77,8 \%)$ dessas mulheres tiveram estádio clínico alto (Tabela 1).

Destaca-se a perda de acompanhamento em 33 $(22,1 \%)$ casos avaliados, principalmente, entre os classificados com estádio avançado do câncer de mama.

Com relação à retirada dos linfonodos, 120 (73\%) mulheres foram submetidas à dissecção axilar completa (Tabela 2).

A quantidade do GP baixo prevaleceu em 50 $(61,7 \%)$ dos casos de câncer de mama classificados como estádio baixo, e o GP alto em 34(87,2\%) daqueles considerados estádio alto (Tabela 2).

Ao diagnóstico imuno-histoquímico 84 (60,4\%) dos casos apresentaram tumores positivos para o 
Análise descritiva e de associação entre as variáveis: C-erbB-2, RhE e RhP com o estádio clínico dos casos de câncer de mama registrados no período de 2005 a 2008 em Aracaju.

\begin{tabular}{|c|c|c|c|c|c|c|c|}
\hline \multirow{3}{*}{ Variável } & \multicolumn{4}{|c|}{ Estadiamento do câncer } & \multirow{2}{*}{\multicolumn{2}{|c|}{ Total }} & \multirow{3}{*}{$p^{*}$} \\
\hline & \multicolumn{2}{|c|}{ Baixo } & \multicolumn{2}{|c|}{ Alto } & & & \\
\hline & $n$ & $\%$ & $\mathrm{n}$ & $\%$ & $\mathrm{n}$ & $\%$ & \\
\hline \multicolumn{8}{|c|}{ Percentual do GP } \\
\hline Baixo & 50 & 61,7 & 31 & 38,3 & 81 & 67,5 & $<0,001$ \\
\hline Alto & 5 & 12,8 & 34 & 87,2 & 39 & 32,5 & \\
\hline \multicolumn{8}{|l|}{ C-erbB-2 } \\
\hline Positivo & 7 & 29,2 & 17 & 70,8 & 24 & 18,9 & 0,026 \\
\hline Negativo & 56 & 54,4 & 47 & 45,6 & 103 & 81,1 & \\
\hline \multicolumn{8}{|l|}{ RhE } \\
\hline Positivo & 49 & 58,3 & 35 & 41,7 & 84 & 60,4 & 0,011 \\
\hline Negativo & 20 & 36,4 & 35 & 63,6 & 55 & 39,6 & \\
\hline \multicolumn{8}{|l|}{ RhP } \\
\hline Positivo & 44 & 60,3 & 29 & 39,7 & 73 & 52,5 & 0,008 \\
\hline Negativo & 25 & 37,9 & 41 & 62,1 & 66 & 47,5 & \\
\hline
\end{tabular}

* teste qui-quadrado para associação; GP=gânglios positivo; C-erbB-2 = marcador tumoral; RhE= receptor hormonal de estrogênio; $\mathrm{RhP}=$ receptor hormonal de progesterona.

receptor hormonal de estrogênio (RhE), 73 (52,5\%)para o receptor hormonal de progesterona $(\mathrm{RhP})$ e $24(18,9 \%)$ apresentavam positividade para o marcador C-erbB-2.

Quanto ao marcador e aos receptores hormonais houve associação com o grau de estadiamento. $\mathrm{O}$ C-erbB-2 manifestou-se negativo em $56(54,4 \%)$ dos casos de estádio baixo e positivo em $17(70,8 \%)$ daqueles considerados estádio alto. Já o receptor hormonal de estrogênio mostrou uma associação positiva, entre os casos estudados, como fator de proteção, visto que $49(58,3 \%)$ dos casos com estádio baixo eram positivos e $35(63,6 \%)$ do estádio alto eram negativos. Já o receptor hormonal de progesterona apresentou uma associação positiva entre $44(60,3 \%)$ dos casos de estádio baixo e negativa em $41(62,1 \%)$ dos de estádio alto, conforme Tabela 2.

No presente estudo $139(84,24 \%)$ prontuários continham informações sobre a análise imuno-histoquímica dos receptores hormonais, um maior quantitativo de positividade apareceu em estádios baixos e negatividade em estádios altos.
Dos 130 prontuários que continham informação sobre o C-erbB-2, apenas 24 (18,9\%) apresentavam superexpressão para o marcador. A positividade para o C-erbB-2 apareceu em 17 (70,8\%) dos nódulos em estádios altos, ou seja, avançados.

Verifica-se na Tabela 3 que a proporção de casos positivos e negativos para o C-erbB-2, RhE e RhP para as mulheres vivas sem evidências de tumor são, significativamente, diferentes, ainda que a proporção de negativos tenha sido maior no C-erbB-2 $(82,8 \%)$ e a dos positivos, maior no $\operatorname{RhE}(68,3 \%)$ e $\mathrm{RhP}$ $(60,6 \%)$. Para o grupo de mulheres vivas com evidência de tumor, não houve diferença significativa na proporção de casos positivos C-erbB-2 como para o RhE e RhP. Mesmo sendo observadas as diferenças percentuais, verifica-se que o $p$-valor do teste de comparação de proporção não foi significativo para o C-erbB-2, RhE e RhP, indicando que a proporção de casos positivos nos grupos de mulheres que vivem sem evidência de tumor e proporção de casos positivos no grupo de mulheres vivas com evidência de tumor é a mesma. 
Proporções de positivos e negativos segundo a situação da paciente para o marcador tumoral e receptores hormonais de estrogênio e progesterona dos casos de câncer de mama registrados no período de 2005 a 2008 em Aracaju.

\begin{tabular}{|c|c|c|c|c|c|}
\hline \multirow{2}{*}{ Situação } & \multicolumn{2}{|c|}{ Positivo } & \multicolumn{2}{|c|}{ Negativo } & \multirow{2}{*}{$p^{*}$} \\
\hline & $\mathrm{n}$ & $\%$ & $\mathrm{n}$ & $\%$ & \\
\hline \multicolumn{6}{|l|}{ C-erbB-2 } \\
\hline Vive sem evidência de tumor & 16 & 17,2 & 77 & 82,8 & 0,460 \\
\hline Viva com evidência de tumor & 3 & 33,3 & 6 & 66,7 & \\
\hline \multicolumn{6}{|l|}{ RhE } \\
\hline Vive sem evidência de tumor & 71 & 68,3 & 33 & 31,7 & 0,784 \\
\hline Viva com evidência de tumor & 6 & 66,7 & 3 & 33,3 & \\
\hline \multicolumn{6}{|l|}{ RhP } \\
\hline Vive sem evidência de tumor & 63 & 60,6 & 41 & 39,4 & 0,952 \\
\hline Viva com evidência de tumor & 5 & 55,6 & 4 & 44,4 & \\
\hline
\end{tabular}

\section{Discussão}

O estudo ratifica o que é descrito na literatura quanto à morfologia predominante do câncer de mama, pois a maioria dos tumores é classificada como CDI, variando de 47 a $90 \%, 15$ enquanto o CLI varia de 5 a $15 \% .4$

Os tumores classificados como estádio alto ocorreram em maior frequência, sendo de pior prognóstico. Em estudo, realizado nos Estados Unidos foram analisados mais de 12.000 casos de câncer, observando-se que $7,9 \%$ das mulheres tiveram diagnósticos nos estádios IIIC e IV. Esse resultado, também, foi observado neste estudo, já que a frequência de tumores classificados como IIIC e IV foi de 7,34\%.15

Ainda quanto ao estadiamento, é importante destacar que $52(37,34 \%)$ mulheres foram diagnosticadas com estádio III, tendo, possivelmente, diminuída a sobrevida em cinco anos, já que o risco de óbito nesse estádio pode ser de 7,18 vezes maior em relação ao estádio I. 16

Estudos na África identifica estádios III e IV em 70 a $90 \%$ dos casos, valores muito superiores àqueles observados nesse estudo, evidenciando assim, que a descoberta precoce do câncer de mama ainda se encontra deficiente em países subdesenvolvidos ou em desenvolvimento. Nestes países, quando as mulheres têm acesso ao diagnóstico a neoplasia mamária encontra-se avançada, com poucas escolhas de tratamento adjuvante e piores prognósticos. 17

Em relação ao tratamento cirúrgico, a indicação de uma cirurgia mais radical deve ser realizada em estádios avançados por apresentarem maiores dimensões do tumor primário e presença significativa de linfonodos comprometidos. 18 Dentre os casos estudados, a determinação do tipo de cirurgia não teve relação com estádio clínico na maioria dos casos, predominando a mastectomia como modalidade cirúrgica aplicada. É importante destacar que $42(57,5 \%)$ mulheres com estádio baixo foram submetidas a mastectomia, ao invés da técnica cirúrgica de conservação da mama, que é eficaz para os casos de neoplasia mamária nos estádios I e II, principalmente, quando associada à radioterapia. Essa técnica tem como principal objetivo melhorar a qualidade de vida das mulheres com câncer de mama, em termos de imagem corporal e ajustamento social e psicológico. 18

A associação da dissecção axilar completa ao tratamento cirúrgico permite obter maiores informações para definir o estadiamento, o controle local da doença e o planejamento da terapia sistêmica. ${ }^{19}$ Porém, sua aplicação é discutida, pelo fato de que em muitos casos não existe um comprometimento severo desses linfonodos. Já a biopsia do LS é um método que está diretamente ligado à menor morbidade, melhor preservação da função do braço e melhor qualidade de vida das pacientes se comparadas àquelas que foram submetidas à 
dissecção axilar completa. 10

Quanto aos marcadores tumorais avaliados pela imuno-histoquímica, os receptores hormonais de estrógeno (RhE) e os de progesterona (RhP) são importantes para avaliação e determinação do tratamento para o câncer de mama. Após a intervenção cirúrgica esses receptores são investigados de acordo com sua expressão no câncer, com resultados de positividade ou negatividade, servindo de orientadores para indicação da linha terapêutica de melhor eficácia. A positividade para os dois receptores, geralmente, está relacionada a uma melhor resposta ao tratamento e a um melhor prognóstico, com ou sem o uso de tamoxifeno. ${ }^{9}$

Também, é indicativo de bom prognóstico no câncer de mama, a identificação de associação relevante entre tumores positivos para os receptores hormonais e tumores menores que dois centímetros. 20

Em se tratando do marcador C-erbB-2, os achados deste estudo diferem dos dados encontrados na literatura, que afirmam a sua ocorrência, geralmente, em cerca de $20 \%$ de todos os cânceres de mama e em $25 \%$ dos CDI. 21 Todavia, essa informação merece ser melhor investigada devido à impossibilidade de alguns casos terem sido avaliados, devido à pequena amostra.

Apesar do marcador C-erbB-2 não influenciar no estadiamento e ser um aspecto que revela um pior prognóstico, a sua positividade na maioria dos nódulos com estádio avançado evidencia um percentual elevado e semelhança com os resultados de estudo desenvolvido por Herchenhorn et al. ${ }^{21}$ que encontraram $48 \%$ de casos positivos para o C-erbB-2, em pacientes estádio clínico IIIA e IIIB. De forma similar os autores citados 21 concluíram que a positividade para C-erbB-2 estava, diretamente, relacionada ao número de linfonodos axilares comprometidos, confirmando, novamente, a relação entre a positividade para o marcador C-erbB-2 e um alto grau de estadiamento, já que esse é diretamente proporcional ao maior número de linfonodos comprometidos.

Também vale ressaltar que as mulheres com câncer de mama que apresentam C-erbB-2 positivo, além de exibirem uma correlação significativa com a presença dos linfonodos axilares, tendem a desenvolver metástases, apresentam alto risco de recidiva precoce e menor sobrevida que aquelas com o oncogene negativo.

\section{Referências}

1. Abreu E, Koifman S. Fatores prognósticos no câncer da mama feminina. Rev Bras Cancerol. 2002; 48: 113-31.
Além disso, identificou-se que existe associação da situação atual da paciente, ou seja, do seguimento, com as demais características que abrange o câncer de mama como: a apresentação de mais casos com expressão negativa do marcador C-erbB-2 e de positividades dos receptores hormonais, além, de menos casos de positividades dos linfonodos avaliados.

Entre as mulheres vivas com evidência de tumor, identificou-se que não existe diferença entre o número de positivos e negativos dos marcadores tumorais.

A relação do estádio com a situação atual da mulher reforça a importância da detecção precoce do câncer de mama para redução da mortalidade, aumento do tempo de sobrevida e qualidade de vida. A detecção da neoplasia em estádios avançados pode aumentar o risco de a mulher necessitar de tratamentos mais agressivos, como a mastectomia e tratamento adjuvante estendido, com o uso de várias substância tóxicas, gerando importante morbidade psicológica e redução na qualidade de vida. Além disso, o tratamento tem maior custo e menor êxito. 22,23

O fato de o carcinoma ductal invasivo, sobressair-se entre os tumores avaliados, evidencia uma população acometida em grande percentual por tumores agressivos. 6 Pelo sistema TNM entre os casos avaliados o câncer de mama localmente avançado (IIB, IIIA, IIIB e IIIC) respondeu pela maioria das neoplasias, demonstrando o quão tardiamente ocorreram o diagnóstico do nódulo entre as mulheres do estudo, já que responderam por (48\%) casos. Como consequência a maioria das mulheres foram submetidas à mastectomia independente do estadiamento clínico, e a maioria das mulheres vivas sem evidência da neoplasia foram aquelas que tiveram o estádio clinico baixo. Dessa forma, o estudo ratifica a importância das ações de detecção precoce para o câncer de mama nos serviços de atenção básica de Sergipe para a redução da mortalidade, morbidade e, melhora da sobrevida e qualidade de vida entre as mulheres que forem acometidas por essa neoplasia.

É de grande valia a realização de novos trabalhos que estudem as associações entre as características que influenciam sobre o curso clínico, tratamento e prognóstico de pacientes com câncer de mama, que venham a subsidiar a elaboração e implementação de estratégias de controle e assistência a esta neoplasia.

2. Thuler LC. Considerações sobre a prevenção do câncer de mama feminino. Rev Bras Cancerol. 2003; 49: 227-38. 
3. Brasil. Ministério da Saúde. Instituto Nacional de Câncer: estimativa da incidência de câncer para 2010 no Brasil e nas cinco regiões; 2010. [acesso em 4 fev 2010]. Disponível em: http://www.inca.gov.br/estimativa/2010/.

4. Alvarenga M. Tumores da mama. In: Billis AVJ, editor. Patologia diagnóstica de tumores. Campinas: Impressão Digital do Brasil Gráfica e Editora Ltda; 2004. p. 205-98.

5. Harvey JA. Unusual breast cancers: useful clues to expanding the differential diagnosis. Radiol. 2007; 242: 683-94.

6. Ashida A, Fukutomi T, Tsuda H, Akashi-Tanaka S, Ushijima T. Atypical medullary carcinoma of the breast with cartilaginous metaplasia in a patient with BRCA1 germline mutation. Jpn J Clin Oncol. 2000; 30: 30-2.

7. Biazus J. Tipos de Câncer. 2005. [acesso em 16 out 2009]. Disponível em http://www.jorgebiazus.com.br/tiposcancer. htm.

8. Brasil. Ministério da Saúde. Secretaria de Assistência a Saúde. Instituto nacional do câncer. Bases técnicas para autorização de procedimentos de alta complexidade (apaconcologia); 2001. [acesso em 24 jul 2009]. Disponível em: http://dtr2001.saude.gov.br/sas/decas/manualsia-susonco.htm.

9. Brasil. Ministério da Saúde. Secretaria de Assistência a Saúde. Instituto nacional do câncer. Controle do câncer de mama. Documento do consenso; 2004. [acesso em 1 nov 2009]. Disponível em: http://www1.inca.gov.br/ publicacoes/Consensointegra.pdf

10. Giuliano AE, Hunt KK, Ballman KV, Beitsch PD, Whitworth PW, Blumencranz PW, Leitch AM, Saha S, McCall LM, Morrow M. Axillary dissection vs no axillary dissection in women with invasive breast cancer and sentinel node metastasis: a randomized clinical trial. JAMA. 2011; 305: 569-75.

11. Dhingra K, Hortobagyi GN. Critical evaluation of prognostic factors. Semin Oncol. 2006; 23: 436-45.

12. Moraes AB, Zanini RR, Turchiello MS, Riboldi J, Medeiros LR. Estudo da sobrevida de pacientes com câncer de mama atendidas no hospital da Universidade Federal de Santa Maria, Rio Grande do Sul, Brasil. Cad Saúde Pública. 2006; 22: 2219-28

13. Palmeira H, Naidu S, Juaçaba S, Ferreira MV, Rabenhorst $\mathrm{SH}$. Características anatomopatológicas e dados epidemiológicos de pacientes com câncer de mama submetidas a

Recebido em 11 de maio de 2011

Versão final apresentada em 28 de dezembro de 2011

Aprovado em 30 de janeiro de 2012 tratamento cirúrgico na Maternidade-Escola Assis Chateaubriand. Rev Bras Mastol. 2002; 12: 34-44.

14. Oliveira MMC, Torresan C, Oliveira SFV, Torrezan GT, Abuázar CS, Lima RS, Urban CA, Cavalli LR, RIbeiro EMSF, Cavalli IJ. Epidemiologia do câncer de mama em pacientes do Sul do Brasil. BEPA. Bol Epidemiol Paulista (Online). 2009; 6: 1

15. Barry J, Breen N. The importance of place of residence in predicting late-stage diagnosis of breast or cervical cancer. Health Place. 2005; 11: 15-29.

16. Schneider IJC, d'Orsi E. Sobrevida em cinco anos e fatores prognósticos em mulheres com câncer de mama em Santa Catarina, Brasil. Cad Saúde Pública. 2009; 25: 1285-96.

17. Fregene A, Newman LA. Breast cancer in sub-Saharan Africa: how does it relate to breast cancer in AfricanAmerican women? Cancer. 2005; 103: 1540-50.

18. Guedes JM, Macéa JR, Rinaldi JF.Qualificação dos linfonodos axilares obtidos em cirurgias para câncer na mama e sua correlação com dados terapêuticos e variáveis epidemiológicas. Rev Bras Mastol. 2004; 14: 135-41.

19. Yang SH, Yang KH, Li YP, Zhang YC, He XD, Song AL, Tian JH, Jiang L, Bai ZG, He LF, Liu YL, Ma B. Breast conservation therapy for stage I or stage II breast cancer: a meta-analysis of randomized controlled trials. Ann Oncol. 2008; 19: 1039-44.

20. Eisenberg A, Koifman S. Câncer de mama: marcadores tumorais: revisão da literatura. Rev Bras Cancerol. 2001; 47: $377-88$.

21. Herchenhorn D, Rezende LM, Thuler LC, Maia RC, Medina M, Costa MDC. Quimioterapia neoadjuvante em câncer de mama localmente avançado: análise imuno-histoquímica é preditiva da resposta à quimioterapia. Rev Bras Cancerol. 2000; 46: 163-71.

22. Unger-Saldaña K, Infante-Castañeda C. Delay of medical care for symptomatic breast cancer: a literature review. Salud Publica Mex. 2009; 51 (Suppl. 2): S270-85.

23. Anderson B, Cheng-Har Y, Ramsey S, Bengoa R, Braun S, Fitch M, Groot M, Sancho-Garnier H, Tsu VD. El cáncer de mama en los países con recursos limitados: sistemas de atención de salud y políticas públicas. Breast J 2007; 13 (Suppl. 1): S62-82. 